\title{
Oncogene-dependent Tumor Suppression: Using the Dark Side of the Force for Cancer Therapy
}

\author{
G.I. Evan, M. Christophorou, E.A. Lawlor, I. Ringshausen, J. Prescott, T. Dansen, \\ A. Finch, C. Martins, AND D. Murphy \\ Cancer Research Institute and Department of Cellular and Molecular Pharmacology, \\ University of California, San Francisco, California 94143-0875
}

\begin{abstract}
Cancers arise by an evolutionary process that involves the protracted acquisition by somatic cells of suites of interlocking mutations that uncouple proliferation, survival, migration, and damage responses from the mechanisms (selective pressures) that normally restrain or restrict them in time and space. The relative rareness of cancer cells within the soma, in the face of huge numbers of available cell targets, substantial rates of mutation, and an abundance of proto-oncogenes and tumor suppressor gene targets, indicates that the evolutionary space available to incipient tumor cells is highly restricted. The principal way in which this is achieved is through intrinsic tumor suppression pathways - innate growth arrest and apoptotic programs that fulfill an essentially analogous functional role to checkpoints in the cell cycle machinery by antagonizing the tumorigenic potential of oncogenic mutations. Using switchable transgenic and knockin mouse models, it is possible to identify these various tumor suppressor programs and establish where, when, how, and why they act to forestall neoplasia in each tissue type and, consequently, how and why their failure leads to cancer.
\end{abstract}

Natural selection works on any replicating biological entity in which genetic diversity arises. Consequently, the lifestyle of long-lived, physically large organisms with regenerative potential like vertebrates encompasses a permanent tension between the forces of selection working at the level of the organism to foster reproductive fitness, and the forces of selection at the level of somatic cells, fostering outgrowth of lethal neoplastic clones. Most metazoans sidestep the entire cancer problem by handing the whole business of organismal reproduction over to ephemeral, small body plans in which extensive somatic cell proliferation in the adult is neither necessary nor possible. In contrast, vertebrate genes consign their longterm futures to versatile corpora of potentially traitorous cells. The rarity of cancer in vertebrates, amid the profligacy of normal somatic cell proliferation and tissue remodeling and regeneration, represents a remarkable example of evolutionary footwork.

Clearly, long-lived organisms require the capacity throughout life to maintain self-renewing tissues like superficial epithelia, immune and hematopoietic systems, and to repair damage. Hence, many somatic cell types must be able to engage, with ease, all the machinery necessary for reconstruction of tissues, including the capacity to expand themselves and to remodel adjacent stroma, vasculature, and accessory cells while keeping infection at bay through proactive establishment of the inflammatory and immune responses. On the other hand, too easy an engagement of such powerful regenerative programs would appear to leave them prey to subversion by mutation, with disastrous neoplastic consequences, given the appreciable mutation rate in somatic cells. The rarity of the cancer cell indicates that, somehow, vertebrate cells have squared the regenerative circle by making the programs required for tissue expansion and remodeling both easy to activate, in the right place at the right time, and at the same time virtually impossible to subvert through mutation. Arguably, this "achievement" is the cornerstone of cancer-free vertebrate longevity (Green and Evan 2002).

In retrospect, the first clue to how vertebrates solve this conundrum came in the early 1980s with the discovery of oncogene cooperation. These seminal observations indicated that individual oncogenic mutations, such as activation of Ras or Myc, or of E1A or E1B, were "insufficient" for phenotypic transformation of normal fibroblastic cells (Land et al. 1983; Van den Elsen et al. 1983). However, the nature of this insufficiency proved elusive. A common interpretation was that no single individual oncogenic lesion was able to engage the full suite of diverse biological programs necessary for oncogenesis. In this scenario, oncogenes cooperate in an additive way by providing elements missing from each other. In the early 1990s, however, the growing appreciation that individual oncogenic mutations can be potent triggers of growth suppression, senescence, and cell death suggested an alternative explanation for oncogene cooperation. According to this idea, the insufficiency of individual oncogenes is because their powerful abilities to orchestrate many, perhaps all, aspects of malignancy are thwarted by their inbuilt tumor suppressor mechanisms. Oncogenes cooperate when they reciprocally gate each other's intrinsic tumor suppressor functions (Fig. 1B) - in effect, individual (proto)oncogenes lie as nodes in networks rather than on linear, cause-and-effect pathways, and specific information is conveyed by the network as a whole rather than parsed through separate modular pathways. From a micro-evolutionary perspective, the great power of the network scenario is that the innate growth-suppressor functions attached to individual nodes in the network effectively mask any immediate selective advantage that might be conferred on a cell from mutating that node - effectively converting it into a potent selective disadvan- 

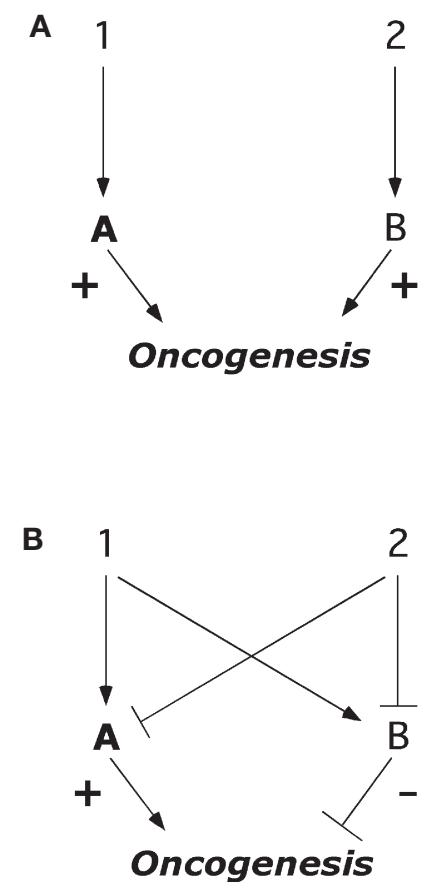

Figure 1. Alternative models describing how signals cooperate to drive malignant transformation. (A) Transformation requires the additive cooperation of two signaling modules, each alone insufficient to drive all the necessary programs required for transformation. (B) Transformation requires the simultaneous activation of two nodes within a network. Neither node is alone sufficient because each node acts both positively and negatively on the transformed phenotype. Only when both nodes are triggered together do the positive effects of each node gate the negative effects of the other, uncovering the transforming potential inherent in each node.

tage. Subversion of the network through mutation becomes almost impossible because individual mutations obligatorily trigger a shutdown of the very biological response that they also activate. Hence, individual mutations are unstable and cannot accumulate one by one, effectively stalling natural selection at the somatic level and so quelling the risk of neoplasia. On the other hand, when $\mathrm{A}$ and $\mathrm{B}$ are activated together in a normal cell responding to appropriate trophic and growth factors (signals 1 and 2 ), the negative impact of $A$ is gated by $B$ and vice versa, unlocking the full potential of the cell's growth, proliferative, angiogenic, and tissue-remodeling programs (Fig. 1B).

Arguably, the most important contra-predictions of the linear versus network models for oncogene interaction pertain to the complexity of tumors and the treatment of cancers. In a linear signaling framework, individual aspects of cell behavior are handled by discrete signaling modules, each of which has to be subverted through the long and protracted accumulation of cognate mutations in each module in order to assemble the wherewithal for the cell to become malignant. Consequently, cancer cells are complex ensembles of mutations, highly polymorphic in nature, and requiring the targeting of multiple pathways to achieve therapeutic efficacy. In contrast, the network idea would suggest that cancer cells are driven and underpinned by a small set of interlocking, interdependent molecular lesions and that the apparent complexity of the neoplastic phenotype merely reflects the highly pleiotropic nature of these underlying lesions. If this is true, then targeting such pivotal lesions would have a powerful therapeutic impact on established cancers.

Determining which of these two models is correct is complicated in human tumors because of their high level of genomic instability, at least in part arising from processes such as telomere erosion that are secondary to the activation of oncogenes or loss of tumor suppressor genes which presumably initiate and drive early neoplastic clonal expansion. The effect of this is to introduce a large level of mutagenic noise that obscures any underlying causal molecular lesions. For this reason, the laboratory mouse, with its outsized telomeres and its experimental tractability, offers many advantages. By installing defined oncogenic lesions, alone and in combination in specific tissues, it is then possible to evaluate how and why such lesions promote neoplasia. In recent years, a further refinement has been the addition of acutely switchable systems with which to toggle oncogenes between normal and activated or tumor suppressor genes between functional and nonfunctional states. This latter refinement has proven critical for assessing the immediate, often growthinhibitory impact of acute oncogene activation or tumor suppressor ablation, and how this leads to the eventual onset of malignancy.

\section{MYC-A PARADIGM FOR INTRINSIC TUMOR SUPPRESSION}

Against this backdrop, the Myc oncoprotein is a paradigm for the complexities dictating selection for oncogenic traits in cancer. Myc is a basic helix-loophelix-leucine zipper (bHLH-LZ) transcription factor encoded by the c-myc proto-oncogene. It is generally assumed that Myc exerts its protean biological effects through modulation of target genes, some genes being repressed by Myc while others are activated. Most Myc-induced genes have a consensus CACGTG E-box element in their promoters which is recognized by Myc in partnership with its heterodimeric bHLH-LZ partner Max. In Myc-repressed genes, E-box elements are often absent, and the precise recognition element is less well-defined. Upon binding, Myc:Max exhibits contextual interactions with various components of the basal transcriptional machinery. For example, Myc recruitment of TRAAP correlates with transcriptional activation, whereas Myc interaction with the SP-1 and Miz-1 zinc finger proteins mediates its down-regulation of the $p 21^{\text {cipl }}$ and $p 15^{i \text { in } 4 b}$ promoters. More generally, Myc acts as a network node that orchestrates the wide variety of diverse biological processes required for proliferation of somatic cells. These include both cell-intrinsic processes such as cell growth, biogenesis, metabolism, and proliferation, telomere maintenance, DNA damage and repair; and social processes that coordinate and integrate cell expansion with the somatic environment, such as cell-stromal interactions, angiogenesis, cell migration and invasion. Importantly, many of the biological consequences of Myc activity are indirect and conditional consequences of the 
transcriptional programs it executes-highly dependent on subtle and ephemeral factors such as cell type, status, and local environment. This pleiotropic and contingent nature of Myc action makes any simplistic distillation of Myc "function" unrealistic. Rather, it seems that Myc serves to establish an eclectic platform of transcriptional changes that empowers the cell with a wide variety of potential biological options.

In normal cells, expression of Myc is tightly dependent on the actions of appropriate mitogens, essentially restricting its pleiotropic activities to cells in receipt of the appropriate extracellular signals for expansion. In contrast, expression of Myc is deregulated in most cancer cells, either through direct mutation or, more commonly, indirectly through the constitutive activation of upstream signaling pathways. This is consistent with the eclectic role played by Myc in promoting cell expansion, which makes Myc a perfect target, at least in principle, for subversion by oncogenic mutation. However, powerful counterselective mechanisms serve to greatly diminish the immediate selective advantage afforded by Myc activation. The first clue that Myc activation, by itself, is insufficient for deregulated cell proliferation came from the early oncogene cooperation studies of Land et al. These studies indicated that Myc is unable to induce in vitro transformation of primary fibroblasts without the aid of auxiliary lesions such as Ras activation (Land et al. 1983). Together with the significant time lag preceding sporadic emergence of clonal tumors in the target tissues of Myc transgenic mice, they reinforced the notion that Myc alone is insufficient to drive tumorigenesis without the cooperation of additional sporadic oncogenic lesions that bestow other aspects of the neoplastic phenotype. The unexpected discovery of Myc-induced apoptosis (Askew et al. 1991; Evan et al. 1992; Harrington et al. 1994a) offered a potential explanation for this obligate need for cooperating oncogenic lesions by indicating that Myc alone is insufficient for tumorigenesis because it harbors an innate lethality that staunches its neoplastic potential (Evan and Littlewood 1998; Evan and Vousden 2001; Lowe et al. 2004).

Two general models have been proposed to explain the conundrum of how Myc can induce apoptosis when activated by oncogenic mutation but not when activated as part of the normal proliferative process. One idea is that cells can innately discriminate between normal and "hyperproliferative" signals, triggering an apoptotic program only in response to the latter. Indeed, recent studies have indicated that cells do possess an intrinsic threshold buffer which insulates some components of their innate $\mathrm{ARF} / \mathrm{p} 53$ tumor surveillance machinery from levels of Myc activation in normal cells (Zindy et al. 2003). However, it remains unclear to what extent this pathway is involved in determining cell viability/death (see below). An alternative idea is that the choice of whether a Mycexpressing cell should live or die is determined by the dynamic relationship between the cell and its trophic environment. In this "dual pathways" model (Harrington et al. 1994a,b), Myc activation always primes the cellular apoptotic program, but so long as the cell remains in its orthotopic somatic environment, activation of the apo- ptotic program is suppressed by local trophic signals. Only when an incipient tumor cell expands beyond the capacity of the local environment to quell the apoptotic program does cell death emerge as a biological output of Myc activity.

There is abundant in vitro evidence supporting the "dual pathways" idea that availability of survival factors determines the apoptotic proclivity of Myc (see, e.g., Askew et al. 1993; Harrington et al. 1994b; KauffmannZeh et al. 1997; Rohn et al. 1998). However, we know so little about the abundance and nature of endogenous survival factors for any cell type in vivo that there is no way a priori of deducing whether acute activation of Myc in any particular somatic cell type would lead to cell expansion (abundant survival signals) or involution (insufficient survival signals). Therefore, to assess the relevance of Myc-induced apoptosis in limiting Myc oncogenic activity in vivo, we have established a number of transgenic models in which an acutely activatable form of Myc has been targeted to various cell types. To do this, we made use of the MycER ${ }^{\mathrm{TAM}}$ fusion protein in which Myc has been fused to a modified ligand-binding domain of the estrogen receptor. The resulting MycER ${ }^{\mathrm{TAM}}$ protein is completely dependent on continuous provision of 4-hydroxytamoxifen (4-OHT) for Myc function: Consequently, MycER $^{\text {TAM }}$ transgenic mice have reversibly switchable Myc function in their target tissues that can be regulated by systemic administration or withdrawal of 4-OHT.

\section{TUMOR COMPLEXITY_FACT OR FICTION?}

To explore the consequences of Myc activation in a very different type of tissue, we have established a transgenic mouse model in which c-MycER ${ }^{\mathrm{TAM}}$ is targeted to pancreatic $\beta$ cells via the insulin promoter. Although not a common source of human cancers, $\beta$ cells nonetheless possess several inherent advantages as a model tissue for tumorigenesis. Normal $\beta$ cells have an intrinsically low proliferative rate, can be easily targeted using the almost completely $\beta$-cell-specific insulin promoter, have a welldescribed ontogeny and lineage relationship with neighboring endocrine and exocrine pancreatic cells, and, most strikingly, reside in 300-400 discrete islet populations dispersed throughout the pancreas. This latter makes possible the simultaneous observation of hundreds of independent foci following acute Myc activation, allowing discrimination between immanent effects of the transgene (which would be expected to arise synchronously in all islets) and those arising from stochastic secondary events (which would appear sporadically in isolated islets). As expected from the potent oncogenic activity of Myc, acute activation of MycER ${ }^{\mathrm{TAM}}$ in pancreatic $\beta$ cells of $p I n s-M y c E R^{T A M}$ mice triggers abundant entry of quiescent $\beta$ cells into $\mathrm{S}$ phase, which is synchronous throughout the $\beta$-cell populations in each of the 300-400 discrete islets within the pancreas. However, acute Myc activation also triggers profuse apoptosis, which rapidly overwhelms Myc-induced proliferation and leads to the rapid involution of all islets-formal evidence that Mycinduced apoptosis can act as a powerful innate tumor suppressor in cells in vivo (Pelengaris et al. 2002). To test 
directly the importance of apoptosis in limiting Myc oncogenic activity, the same mice were then crossed into a strain in which the apoptosis inhibitor $\mathrm{Bcl}-\mathrm{x}_{\mathrm{L}}$ is constitutively expressed in $\beta$ cells. Activation of MycER ${ }^{\mathrm{TAM}}$ in the $\beta$ cells of these $p I n s-M y c E R^{T A M} \times R I P 7-B c l-x_{L}$ mice triggered immediate and progressive $\beta$-cell expansion, synchronously in all islets (Pelengaris et al. 2002). Moreover, sustained Myc activation in the presence of Bcl- $\mathrm{x}_{\mathrm{L}}$ rapidly led to synchronous acquisition of many traits characteristic of advanced neoplastic lesions, including loss of cell-cell contact, profuse angiogenesis, dysplasia, anaplasia, local invasion, and metastasis within only a few days (Pelengaris et al. 2002). The rapidity and synchrony with which such traits emerge in each of the hundreds of discrete pancreatic islets argue strongly against the need for sporadic secondary events and, instead, indicate that all of these diverse aspects of tissue reorganization that accompany tumor evolution are encoded by activated Myc, requiring only suppression of apoptosis to unmask them. This is consistent with the highly diverse nature of genes identified as Myc targets (see below) and strengthens the intriguing possibility outlined earlier that the complexity of the tumor phenotype, rather than being the unhappy confluence of very many disparate mutations, each responsible for governing a specific neoplastic trait, is the consequence of a quite small number of interdependent mutations, each in key pleiotropic regulators of cell and tissue fate. In the case of Myc and Bcl$2 / \mathrm{Bcl}_{\mathrm{L}}$, the dramatic oncogenic synergy they exhibit arises because $\mathrm{Bcl}-2 / \mathrm{Bcl}-\mathrm{x}_{\mathrm{L}}$ blocks Myc-induced apoptosis while c-Myc overcomes the powerful ability of Bcl-2Bcl- $\mathrm{x}_{\mathrm{L}}$ to suppress cell proliferation (Fig. 2). Thus, neither Myc nor $\mathrm{Bcl}-2 / \mathrm{Bcl}-\mathrm{x}_{\mathrm{L}}$ alone can promote cell expansion, whereas coordinate activation of the two together is sufficient to unlock the full potential of $\beta$ cells

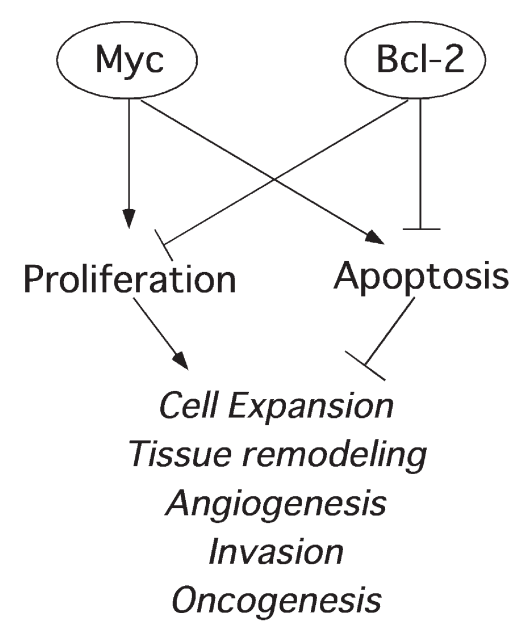

Figure 2. Schematic depiction of the relationship between Myc and $\mathrm{Bcl}-2$ in oncogenesis. Myc drives proliferation, but this is undercut by the dominant role of Myc-induced apoptosis. Bcl-2 suppresses apoptosis, but at the cost of inhibiting cell proliferation. Activation of either alone is an evolutionary cul-de-sac for any cell. However, when Myc is activated together with $\mathrm{Bcl}-2$, Myc overcomes the antiproliferative effect of Bcl-2, and Bcl-2 suppresses the apoptotic action of Myc, allowing activation of cell proliferative programs. to expand, generate requisite neovasculature, and remodel local tissue architecture to cope with that expansion. This interlocking relationship between Myc and $\mathrm{Bcl}-2 / \mathrm{Bcl}-\mathrm{x}_{\mathrm{L}}$ is remarkably similar to that depicted in the interactive network outlined earlier in Figure 1B.

The potent oncogenic synergy between Myc and Bcl-2 raises the issue of whether the only oncogenic lesions that can cooperate with Myc are those that suppress apoptosis. In this context, loss of the ARF or p53 tumor suppressors is especially intriguing. A large number of studies indicate that the ARF/p53 tumor suppressor pathway plays an important role in limiting Myc oncogenic activity and that loss of either ARF or p53 is strongly selected during tumorigenesis (Eischen et al. 1999). Consistent with this, direct ablation of ARF or p53 accelerates tumorigenesis in Myc transgenic mouse models in all tested tissues. Furthermore, Myc activation induces up-regulation of ARF which, in turn, triggers accumulation of p53 (Zindy et al. 1998), and such activation of p53 has been implicated in mediating a component of the Myc-induced apoptotic signal (Hermeking and Eick 1994; Wagner et al. 1994; Zindy et al. 1998). To test directly the roles of ARF and p53 in mediating Myc-induced apoptosis in vivo, pIns-MycER ${ }^{\text {TAM }}$ mice were crossed into either $\mathrm{ARF}^{-/-}$or $\mathrm{p} 53^{-/-}$backgrounds and Myc was activated. In both cases, synchronous eruption of tumors was observed in every islet, essentially phenocopying the tumorigenesis in the pIns-MycER ${ }^{T A M} \times$ RIP7-Bcl- $x_{L}$ mice (Fig. 3). However, close inspection of the dynamics of tumor growth in each case revealed fundamental differences in the mechanism by which tumors formed in each instance. In the pIns-MycER ${ }^{T A M} \times$ RIP7-Bcl- $x_{L}$ mice, Myc induced significant proliferation without any measurable apoptosis (Fig. 4). In contrast, activation of Myc in the absence of ARF induced far more pervasive and sustained proliferation, but this was accompanied by significant cell death (Fig. 4): Indeed, the extent of Myc-induced cell death in pIns-MycER ${ }^{T A M} \times \mathrm{ARF}^{-/}$islets exceeded that in single transgenic $p I n s-M y c E R^{T A M}$ islets at each measured time point. The unexpected conclusion from this analysis is that inactivation of the ARF/p53 pathway promotes Myc oncogenic activity not by forestalling apoptosis but, rather, by enhancing Myc-induced $\beta$-cell proliferation to such an extent that it overwhelms apoptosis.

Two important conclusions can be drawn from such analysis. First, it shows that in addition to its well-described ability to prime the apoptotic program, Myc also triggers a second, discrete, innate tumor suppressor pathway that acts to suppress cell proliferation through activation of $\mathrm{ARF} / \mathrm{p} 53$. As with Myc-induced apoptosis, activation of this antiproliferative program is likely to be extensively modulated by extracellular and social signals. Indeed, there is evidence that ARF expression is, like apoptosis, suppressed by growth and survival factors (Inoue et al. 1999). Nonetheless, elegant experiments by Sherr and colleagues, in which a GFP reporter is inserted into the endogenous $A R F$ locus (Zindy et al. 2003), also indicate that induction of ARF is "insulated" from the low levels of Myc induced during normal mitogenesis by some form of innate buffer that, in effect, discriminates between normal and abnormal levels of Myc. The extent 


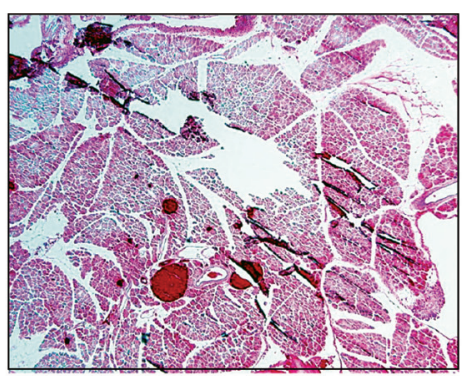

Myc

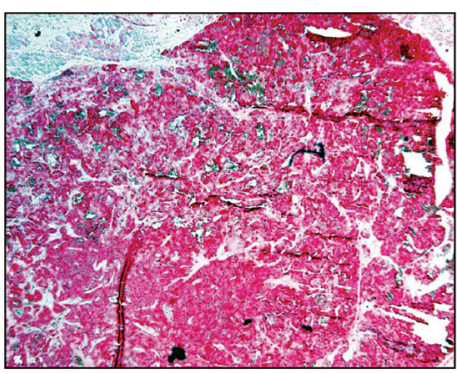

Myc/ARF KO

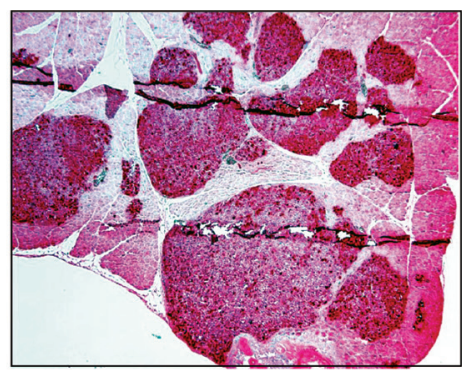

$\mathrm{Myc} / \mathrm{p53} \mathrm{KO}$

Figure 3. Loss of either ARF or $\mathrm{p} 53$ uncovers Myc oncogenic potential in vivo. Myc was activated in $\beta$ cells of $p \operatorname{Ins}-M y c E R^{T A M}$ mice for 21 days (in the absence of ARF, center panel) or 15 days (in the absence of $\mathrm{p} 53$, right panel). In both cases, rapid $\beta$-cell expansion and neoplasia were induced.

to which the apoptotic versus antiproliferative programs act to suppress Myc-dependent oncogenesis, or oncogenesis induced by other dominant oncogenic lesions, is unclear, although it seems plausible that the relative contributions each makes will vary between cell types and with circumstance.

Second, tumor formation requires only that net cell gain exceeds net cell loss. It is therefore possible for secondary oncogenic lesions to cooperate with Myc by either of two distinct mechanisms - through suppression of concomitant apoptosis, as in the case of $\mathrm{Bcl}-\mathrm{x}_{\mathrm{L}}$ expression, or by leaving apoptosis unaffected and enhancing proliferative rate, as in the removal of the intrinsic brake to Myc proliferative potential through inactivation of $\mathrm{ARF} / \mathrm{p} 53$. This conclusion has one important addi- tional implication. Either apoptosis suppression or enhanced proliferation is, alone, sufficient to foster lethal outgrowth of a tumor in combination with activated Myc.

\section{DECONSTRUCTING THE ROLE OF MYC IN TUMOR MAINTENANCE}

Driving relentless cell proliferation without concomitant compensating apoptosis is sufficient for cell expansion, but is it sufficient to drive and maintain tumorigenesis? Classic models of multistage carcinogenesis posit that tumors are the result of the protracted acquisition of multiple mutations, each adding its own suite of traits to the final malignant mix. A need for multiple mutations
Myc alone

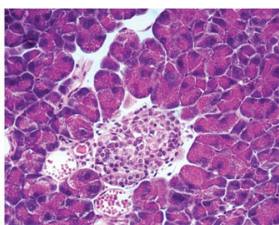

Myc + Bcl- $x_{L}$

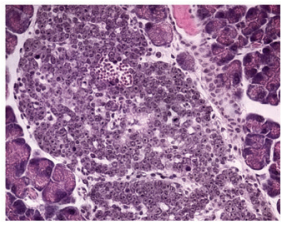

Myc + ARF KO

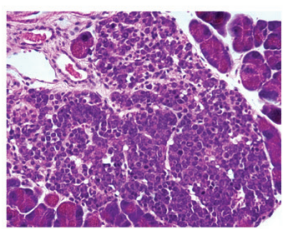

H\&E
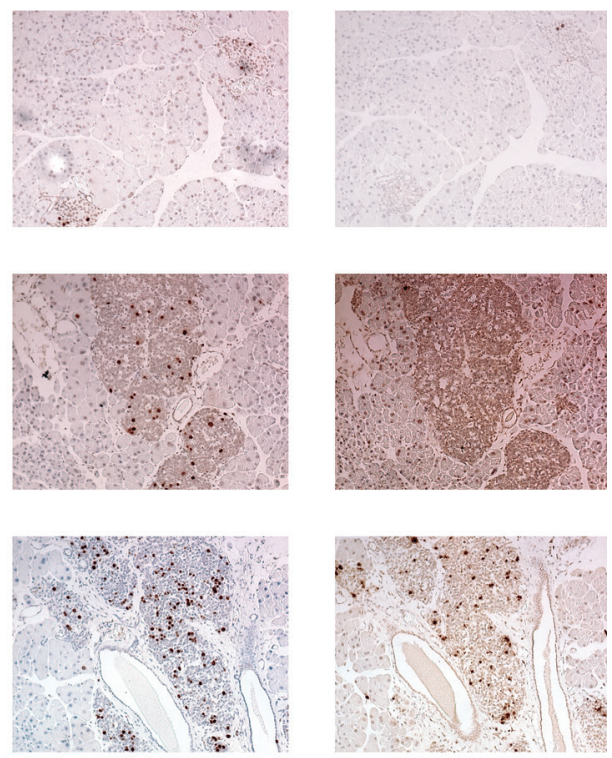

S phase

(BrdU)

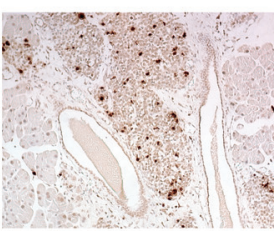

Apoptosis (TUNEL)

Figure 4. Myc-induced tumors can arise by two distinct mechanisms. Myc was activated for 9 days in pancreatic $\beta$ cells, inducing substantial islet involution due to the preponderance of Myc-induced apoptosis (top row). When apoptosis is suppressed, for example, by overexpression of $\mathrm{Bcl}-\mathrm{x}_{\mathrm{L}}$ or loss of the obligate apoptotic protein Bax, Myc activation induces progressive $\beta$-cell/islet expansion characterized by sustained $\beta$-cell proliferation (BrdU) and lack of TUNEL-positive apoptotic $\beta$ cells (middle row). Activation of Myc for 9 days in the absence of ARF also leads to dramatic islet hyperplasia and tumorigenesis. However, this tumorigenesis is characterized by very high levels of proliferation that overwhelm the substantial extent of apoptosis still occurring (bottom row). 
explains both the remarkable rarity of cancer cells within the soma and the complex phenotypes of established tumors, and is lent credibility by the extensive genomic aberrations seen in many human epithelial tumors. On the other hand, just because tumor cells harbor many genetic lesions need not imply that they are all necessary for maintenance of the established tumor. Tumors arise by an evolutionary process, so some mutations may reflect adaptation to selective pressures early in tumor evolution that are irrelevant at later times. Yet others may be irrelevant bystanders that become co-amplified, co-deleted, or co-mutated during the epochs of genome instability known to occur during tumor evolution due to telomere erosion or inactivation of DNA maintenance/repair genes. Importantly, only those mutations that are continuously required for the maintenance of established tumors will be targets for function-based cancer therapies. Consequently, there is a pressing need to filter out of the extensive mutagenic noise in cancer cells those mutations that are causally responsible for tumor maintenance. Switchable oncogenic models offer a unique way to assess directly, in vivo, how far defined oncogenic lesions (alone and in combinations) can drive normal somatic cells along the pathway to true malignancy and whether, once there, those same lesions are required to maintain an established tumor.

In this context, it is especially intriguing that sustained activation of MycER ${ }^{\text {TAM }}$ in $\beta$ cells under circumstances where Myc-induced apoptosis is overcome by Mycinduced proliferation, whether this is by direct blockade (as in overexpression of Bcl- $\mathrm{x}_{\mathrm{L}}$ ) or overwhelmed (as in loss of ARF or p53), leads seamlessly to synchronous transformation of all islets into large, dysplastic, angiogenic, and invasive $\beta$-cell masses. The rapidity (days) and synchrony with which each of the several hundred discrete islets transforms into tumors precludes requirement for any secondary mutations to orchestrate these pleiomorphic traits and, instead, indicates that all these attributes of advanced neoplasia - angiogenesis, dysplasia, anaplasia, local invasion, and metastasis - are orchestrated by Myc, becoming overt only under circumstances where apoptosis is sufficiently overwhelmed to allow progressive cell expansion. The rapid and complete regression of all the tumors upon subsequent deactivation of Myc confirms the notion that all these diverse aspects of the tumor phenotype are directly and continuously dependent on sustained Myc function. Although such findings suggest that Myc may be a critical node in the maintenance of the intertwined network of aberrant signaling pathways that sustain established tumors, it is difficult to extrapolate from artificial, Myc-induced transgenic tumors to spontaneous human tumors where Myc activation may be secondary (in both function and timing) to other oncogenic lesions or to the many tumors where Myc appears not to be mutated itself but acting as a passive conduit for upstream oncogenic flux. Such concerns can only be addressed by construction of wholly new types of switchable mouse models in which the activities of endogenously expressed proto-oncogenes can be ectopically regulated at will.

Nonetheless, the highly pleiotropic nature of Myc suggests that it would make an excellent target for pharma- cological perturbation in cancers. Unfortunately, Myc is a transcription factor, and all evidence indicates that it implements all of its diverse biological functions (tumor promoting and tumor suppressing) through modulation of target genes. Myc interacts with its obligate bHLH-LZ partner Max via an extensive, coiled-coil interactive surface that is difficult to disrupt with small molecules, and with a variegated and redundant ensemble of components within the basal transcription machinery. Currently, such considerations are thought to rule out Myc as a "druggable" target and have, instead, focused attention on defining critical downstream transcriptional targets of $\mathrm{Myc}$, in the hope of finding obligate Myc effectors more amenable to therapeutic intervention. In this context, a "critical" Myc target can be defined as one whose continuous modulation by Myc is required for maintenance of Myc-driven tumors. Unfortunately, the breadth of Myc target genes is huge: Expression microarray or SAGE studies of Myc target genes in lymphocytes or fibroblasts in vitro indicate that hundreds, perhaps over a thousand, genes are directly modulated by Myc (Coller et al. 2000; Guo et al. 2000; Menssen and Hermeking 2002; O'Connell et al. 2003; Zeller et al. 2003). More recent chromatin immunoprecipitation studies have suggested that Myc may be associated with perhaps $5-10 \%$ of all genes (Fernandez et al. 2003; Haggerty et al. 2003; Mao et al. 2003). Even a cursory survey of Myc gene targets indicates an enormously broad range of biological activities encompassing virtually all aspects of the cell, including tissue function, cell growth, proliferation, metabolism, biogenesis, cytoskeleton trafficking, intracellular and intercellular signaling, and tissue organization. The highly pleiotropic nature and large number of Myc target genes support the emerging notion that Myc acts as a contextual regulator of global transcription rather than as a driver of specific biological programs (Orian et al., this volume).

Unfortunately, dissecting out from such a large, diffuse and functionally heterogeneous ensemble of gene targets those that are "critical" for specific aspects of the Myc neoplastic phenotype is extremely difficult. Hitherto, the general approach has been to use an unbiased genomewide approach such as gene expression arrays or chromatin immunoprecipitation to construct a look-up table of Myc target genes and then use human bias, based on preconceived ideas of what genes are important, to filter the candidates down to a "manageable" number that is experimentally testable. Although state-of-the-art, this strategy is an unhappy marriage of objectivity and subjectivity, given the parlous nature of our knowledge of gene functions. In contrast, reversibly switchable transgenic Myc tumor models offer a completely unbiased strategy for deconvoluting the complexity of Myc function, at least with respect to tumorigenesis and tumor maintenance. Following acute Myc activation, it is possible to correlate temporally Myc-induced transcriptional changes in an orthotopic tissue with the cell and tissue changes that accompany tumor progression. Likewise, subsequent deactivation of Myc in established tumors allows temporal correlation of onset of tumor regression with the underlying transcriptional changes that precipitate it. Furthermore, by combining these two analyses, it is possible to 
define an even more limited cohort of Myc target genes whose expression is inversely regulated upon Myc activation (tumor progression) and subsequent deactivation (tumor regression). Since Myc function is continuously required to maintain tumors in these switchable Myc models, this cohort comprises candidate genes required for tumor maintenance (E.A. Lawlor et al., in prep.).

Figure 5 illustrates such a kinetic progression-regression analysis of Myc-responsive genes. For the tumor progression study, MycER ${ }^{\mathrm{TAM}}$ was acutely activated in pancreatic $\beta$ cells of $p I n s-M y c E R^{T A M} \times R I P 7-B c l-x_{L}$ mice by systemic administration of 4-OHT and, at various times thereafter, islets were isolated by laser capture micro-dissection. RNA was then extracted, amplified, and used to probe an Affymetrix U74Av2 mouse gene array. For the regression study, daily 4-OHT administration to $\mathrm{pIns}-M y c E R^{T A M} \times R I P 7-B c l-x_{L}$ mice was stopped after 21 days, and the $\beta$-cell tumors were isolated by laser capture after $0,1,2$, or 3 days. Gene expression was assayed on the same Affymetrix chips. 4-OHT has a tissue halflife of $\sim 30$ hours, so Myc deactivation occurs between 1 and 2 days after 4-OHT surcease.

During both Myc activation and subsequent deactivation, expression of several hundred genes is significantly modulated. Of the genes regulated upon Myc activation,

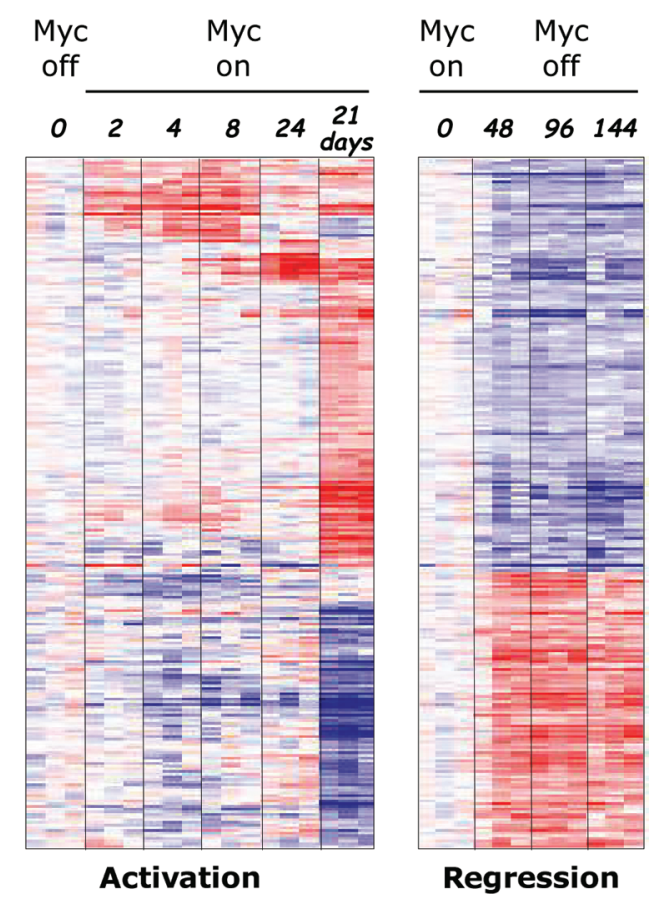

Figure 5. Kinetic analysis of Myc-regulated changes in gene expression in $\beta$ cells during tumor initiation and subsequent regression. Myc was activated in pancreatic $\beta$ cells of $p$ Ins-Myc$E R^{T A M}$ mice, and at $0,2,4,8$, and $24 \mathrm{hr}$ thereafter, RNA was isolated from laser-captured, microdissected islets and analyzed by Affymetrix MG-U74Av2 array. Myc activation was then sustained for 21 days to generate $\beta$-cell tumor RNA (21-day time point). After 21 days, daily administration of 4-OHT was stopped and RNA was isolated from regressing tumors after 2, 4, and 6 days (tumor regression cohorts). SAM analysis identified significantly modulated genes, of which those that were reciprocally modulated during Myc activation and deactivation are shown. Red indicates induction of expression; blue, repression. those whose expression is modulated within 2-4 hours of acute Myc activation are most likely to be direct Myc targets, whereas genes modulated only later ( 24 hours) are more likely the indirect consequences of programs engaged by those early transcriptional targets. Consistent with this, most (75\%) of the genes up-regulated by Myc at either 2 or 4 hours have consensus Myc:Max-binding E-box elements in their promoters, compared with only $30 \%$ of those up-regulated at 24 hours. By comparing the Myc activation and deactivation cohorts at all time points, we can identify 257 candidate tumor maintenance probe sets based on the fact that their expression is inversely modulated following Myc activation (tumor induction) versus subsequent Myc deactivation (tumor regression). Importantly, of these 257, only 11 genes qualify as direct Myc targets (the rest are most likely indirect consequences of Myc action) - a number that is small enough to allow direct experimental validation of each candidate, alone and in combination. Clearly, such functional analyses will be needed to validate the role each plays in maintenance of Myc-dependent tumors. Nonetheless, the approach suggests that rapidly switchable and reversible genetic models, by marrying dynamic changes in cell and tissue biology to the molecular changes that cause them, can be used to filter the diffuse abundance of data generated by genome-wide screens in a completely unbiased way into tractable numbers of candidates for further testing.

\section{PARTNERS IN CRIME-THE ROLE OF p53 AS A COOPERATING ONCOGENIC LESION IN TUMOR MAINTENANCE}

The dramatic oncogenic synergy between deregulated Myc and inactivation of the ARF/p53 pathway attests to the importance of the p53 pathway in restricting Myc's oncogenic proclivities. Similar tumor-suppressive roles for the p53 pathway have been defined for essentially every type of oncogenic mutation, most notably oncogenic mutations of Ras.

p53 appears to have evolved early in metazoan evolution and, since neoplasia is unlikely to have been a major risk factor for such ephemeral progenitor species, it seems most likely that p53 first evolved as a transcriptional integrator of the DNA damage response to preserve genome integrity in germ-line and somatic cells. This ancestral DNA damage response function has been retained in vertebrates and is thought by many to constitute the critical component of p53's tumor-suppressive mechanism. According to this "Guardian of the Genome" idea (Lane 1992), p53 staunches the capacity of mutated or genomically unstable cells to propagate within the soma by implementing either a suicidal or growth-arrest response to genotoxic injury. In addition to this DNA damage response, however, p53 has also evolved the capacity to respond to a variety of non-genotoxic insults - most pertinently, aberrations in proliferative pathways. Thus, activation of multiple oncogenes such as Myc, Ras, E2F, and Wnt trigger a profound p53 response in the absence of any overt or detectable injury to cellular DNA, an attribute that may reflect an evolutionarily more recent 
adaptation to deal with the risk of neoplasia in large, longlived organisms with self-renewing tissues like mammals. As already outlined above, the principal molecular mediator of this growth-deregulation response pathway is the ARF tumor suppressor that acts to stabilize p53 by blocking p53 degradation by its E3-ubiquitin ligase, Mdm-2. Furthermore, p53 is activated by a wide range of other cell stresses that are not obviously genotoxic in nature, including nutrient privation, virus infection, and hypoxia, many of which are likely to be relevant to tumor cells in vivo.

The relative importance of DNA-damage versus growth-deregulatory or other pathways as triggers of p53 tumor-suppressive function during tumorigenesis and tumor evolution remains unclear, mainly because we have little idea as to which of these p53-activating pathways is triggered, or why and when, during the genesis and progression of any tumor type. Hence, defining the temporal requirements for $\mathrm{p} 53$-mediated tumor suppression would shed much needed light on what types of genetic lesions initiate, drive, and maintain cancers in any particular tissue. Importantly, for any therapeutic strategy based around restoring p53 function in cancer cells, it is vital to know whether the signals that activate p53 in tumor cells are transient and episodic or, instead, sustained throughout tumor progression. For example, were p53 to act solely to mediate growth-suppressive responses to hitand-run mutagenic damage in somatic cell DNA, its tumor-suppressive activity would be confined only to cells harboring acute damage, and any therapeutic effect of restoring $\mathrm{p} 53$ function to tumors would be restricted to cancer cells in receipt of ongoing genotoxic injury. In contrast, if p53 is activated by signals that are sustained in tumor cells (for example, deregulated oncogenes, abnormal chromosome content or architecture, nutrient or hypoxic stress), its restoration would presumably exert a therapeutic effect at any time.

Classic gene knockout strategies in mice have, of course, provided important validation of the global significance of p53 in tumor suppression. More recently, conditional knockouts using cre-lox recombinase technology have refined such studies by circumventing the ill-defined and often occult developmental compensation that frequently arises in germ-line knockouts and allowing study of the acute consequences of p53 elision. However, even the latter are limited by the fact that gene inactivation is, typically, far from uniform within the target tissue population and, moreover, is irreversible. This means it is not possible to explore what the consequences of p53 functional restoration, transient or permanent, might be during the course of development, tissue maintenance of tumorigenesis. Therefore, to establish a mouse in which p53 functionality can be reversibly toggled between wild-type and knockout states in tissues in vivo, we turned to the ER ${ }^{\mathrm{TAM}}$ switch technology we had already established in our switchable MycER ${ }^{\mathrm{TAM}}$ transgenic models. In prior in vitro studies, we had already shown the $\mathrm{p} 53 \mathrm{ER}^{\mathrm{TAM}}$ fusion protein to be reliably and tightly regulated by 4-OHT for all measurable aspects of p53 function (Vater et al. 1996). Importantly, p53ER ${ }^{\mathrm{TAM}}$ is not activated by the 4-OHT ligand but, instead, rendered competent to become activated by appropriate p53-activating signals. Hence, a cell expressing p53ER ${ }^{\mathrm{TAM}}$ in place of p53 is effectively p $53^{-/-}$in the absence of 4-OHT ligand and $p 53^{w t}$ in its presence. By inserting the $\mathrm{ER}^{\mathrm{TAM}}$ switch in-frame into the carboxy-terminal exon 11 of the endogenous $p 53$ gene, we generated a mouse expressing p53ER ${ }^{\mathrm{TAM}}$ in place of p53. Expression of p53ER ${ }^{\mathrm{TAM}}$ in these $p 53 E R^{T A M}$ Knockin $(K I)$ mice is controlled by the same orthotopic transcriptional regulatory sequences that normally regulate $p 53$ expression in each tissue in vivo. However, since the expressed p53ER ${ }^{\mathrm{TAM}}$ protein is functionally competent only in the presence of 4-OHT ligand, the $p 53 E R^{T A M} K I$ mouse provides an unparalleled platform for the specific and temporally controlled perturbation analysis of p53 function, both in mouse tissues in vivo and in explanted primary cells derived from such tissues in vitro (Christophorou et al. 2005).

As expected, $p 53 E R^{T A M} K I$ mice in the absence of 4OHT resemble classic p53 knockouts in their high incidence of spontaneous tumors - principally lymphomawhereas their radiosensitive tissues (spleen, thymus, intestinal epithelium) exhibit significant refractoriness to radiation-induced apoptosis (Christophorou et al. 2005). Systemic administration of 4-OHT to $p 53 E R^{T A M} K I$ mice rapidly restores $\mathrm{p} 53$ function in all tissues, reestablishing radiosensitivity in thymus, spleen, and intestinal epithelium in vivo, as well as in cells derived from $p 53 E R^{T A M}$ $K I$ mice in vitro (Fig. 5).

Both normal and neoplastic cells in vitro and in vivo exhibit a marked proclivity to undergo genomic catastrophe in the absence of p53 (Harvey et al. 1993; Fukasawa et al. 1996, 1997). Consequently, tissues of non-4-OHTtreated $p 53 E R^{T A M} K I$ mice acquire an appreciable load of cells harboring genetic defects during the course of embryonic development and postnatal life (Fukasawa et al. 1997). The unresolved damage residing in such aberrant somatic cells might be expected to trigger p53 upon its functional restoration in adult mice, inducing diverse tissue pathologies. Surprisingly, however, restoration of p53 function for the first time to adult mice that have hitherto been devoid of p53 activity induces neither any observable pathology nor detectable expression of p53 target genes in any tissue (Christophorou et al. 2005). One possible explanation for this is that, in the absence of a p53-mediated arrest or apoptotic response, DNA damage or chromosomal defects are rapidly resolved, at least at the biochemical level, such that the p53-activating signal rapidly attenuates. Using the $p 53 E R^{T A M} K I$ mouse model, it is possible to test this hypothesis directly by subjecting animals to a single radiation dose in the absence of p53 function, and then restoring p53 function at various times thereafter. Such studies indicate that the p53-activating signal induced by radiation in signal radiosensitive tissues (thymus, spleen, intestinal epithelium) is, indeed, very transient-decaying to baseline within 48-72 hours of the original insult.

Such studies raise some important questions as to what the triggers are in inchoate tumor cells that activate p53 and suppress the emergence of neoplastic cells within the 
soma. Our experiments reveal that the DNA damage signal activating p53 is fleeting, so unless tumor cells harbor chronic DNA damage, this is unlikely to be other than an episodic trigger of p53, perhaps critical only at the time of exposure to carcinogens or during crisis following telomere erosion. On the other hand, several non-genotoxic triggers can activate p53, and some of these, most notably activated oncogenes, will be persistent features of tumor cells throughout their tortuous evolutionary trajectories.

To investigate directly the role of the DNA damage response in p53-mediated tumor suppression, p53ER ${ }^{T A M} K I$ mice were exposed to a lymphomagenic dose of radiation with $p 53 E R^{T A M}$ nonfunctional, functional during the time of irradiation but not thereafter, or nonfunctional during irradiation but transiently restored at a later time well after the DNA damage response had attenuated. Mice in which $p 53 E R^{T A M}$ was nonfunctional throughout showed no ill effects from the radiation but $100 \%$ mortality from lymphoma by 24 weeks. Mice in which p53 was functional at the time of irradiation exhibited all the expected radiation-induced pathologies, yet $85 \%$ were dead from lymphoma at 24 weeks, indicating only modest tumor suppression. Mice in the third group, in which p53 function was transiently restored at later times, showed no pathology nor measurable activation of p53 target genes in target lymphoid tissues at the time of irradiation, nor at the time of delayed p53 restoration. Nonetheless, $80 \%$ of the mice were alive and well at 24 weeks, indicating profound suppression of tumorigenesis. These data indicate that long after their non-tumorigenic siblings have successfully recovered from acute genotoxic injury, the few incipient tumor cells that have acquired oncogenic mutations still harbor persistent signals which can be engaged by p53 to suppress their outgrowth. Exactly what these signals are remains unclear: However, obvious candidates are mutations that deregulate cell proliferative machinery, since these are known to activate p53 and must be persistent features of tumor progression and maintenance.

\section{p53 AS "GUARDIAN OF GENOME"-SHIVA OR SHIBBOLETH?}

Much has been made recently of the potential for p53 and other tumor suppressors to exhibit "antagonistic pleiotropy" - in effect, an evolutionary compromise in which provision of effective tumor suppression up to reproductive age comes at the cost of eroding post-reproductive life span (Green and Evan 2002; Campisi 2003; Lowe et al. 2004). Studies such as ours are consistent with such ideas and, moreover, raise the disquieting possibility that the DNA damage response, far from being the linchpin of p53-mediated tumor suppression, is instead an "unfortunate" relic of p53's evolutionary origins. "Unfortunate" because the p53-mediated DNA damage response indiscriminately kills or arrests cells with damaged DNA, even though the vast majority of such cells would never become tumors (as evidenced by the clonality of tumors that arise following such genotoxic insults, even in p53-deficient mice). The result of such indiscriminate culling is a wide range of pathologies, includ-
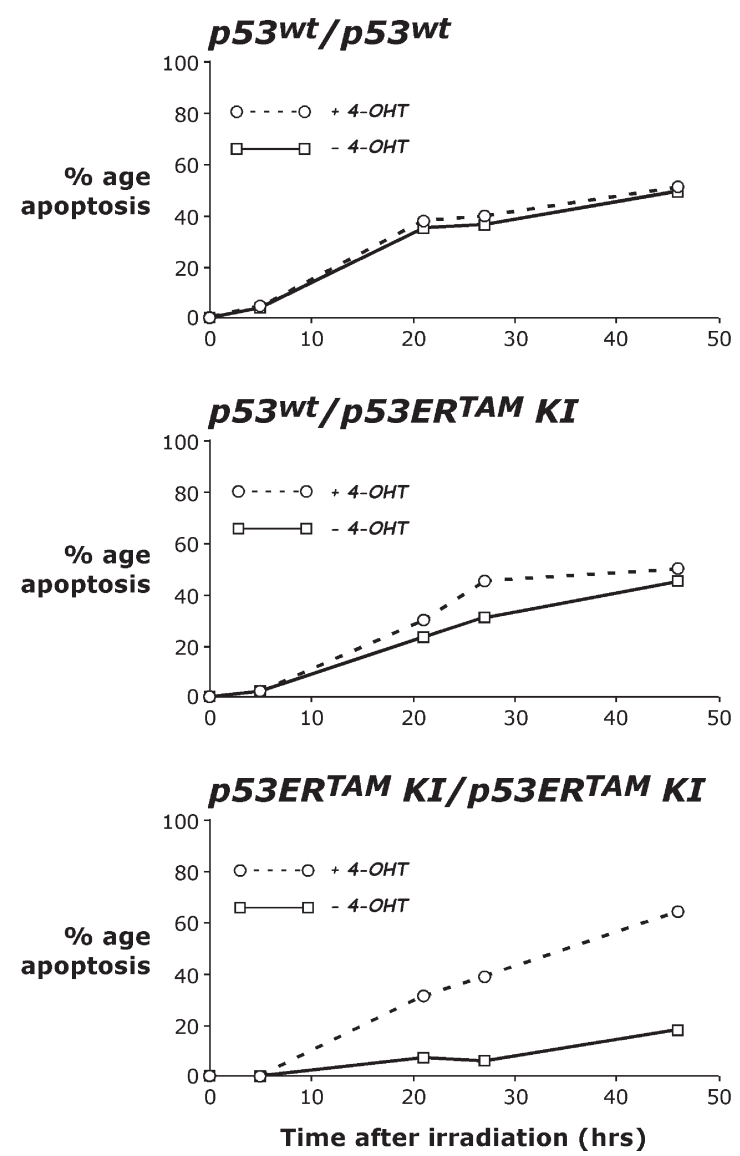

Figure 6. Systemic administration of the $\mathrm{ER}^{\mathrm{TAM}}$ ligand 4-OHT to $p 53 E R^{T A M} K I$ mice restores DNA damage sensitivity to thymocytes. Thymocytes of $p 53^{w t}, p 53^{w t} / p 53 E R^{T A M} K I$ heterozygous, and p53ER ${ }^{T A M}$ homozygous $K I$ mice were irradiated with 3 Gy gamma radiation either in the absence or presence of 4OHT. In $p 53 E R^{T A M} K I$ mice, thymocyte apoptosis occurs only when 4-OHT is present and $\mathrm{p} 53 \mathrm{ER}^{\mathrm{TAM}}$ has been functionally restored.

ing iatrogenic responses to radiation and chemotherapy and, perhaps, through the cumulative attrition of stem cells that encounter damage throughout life, even to organismal aging (Fig. 6).

In contrast to the DNA damage response, however, the response of p53 to growth deregulating mutations is highly specific only for those rare cells that, as a consequence of DNA damage, acquire oncogenic mutations and are therefore likely to evolve into tumors (Fig. 7). Moreover, such oncogenic mutations are likely to be persistent features of tumor cells, throughout their tortuous evolution, and may therefore constitute more efficient triggers of p53 activation than ephemeral DNA damage signals. One radical idea emerging from such considerations is that decoupling p53 from the DNA damage response while retaining the p53 response to growth deregulation might offer the benefits of effective tumor suppression without the demerits of the pathologies associated with acute and chronic DNA damage. Unfortunately, the pathways directly or indirectly linking DNA damage with p53 activation are extremely diverse and re- 


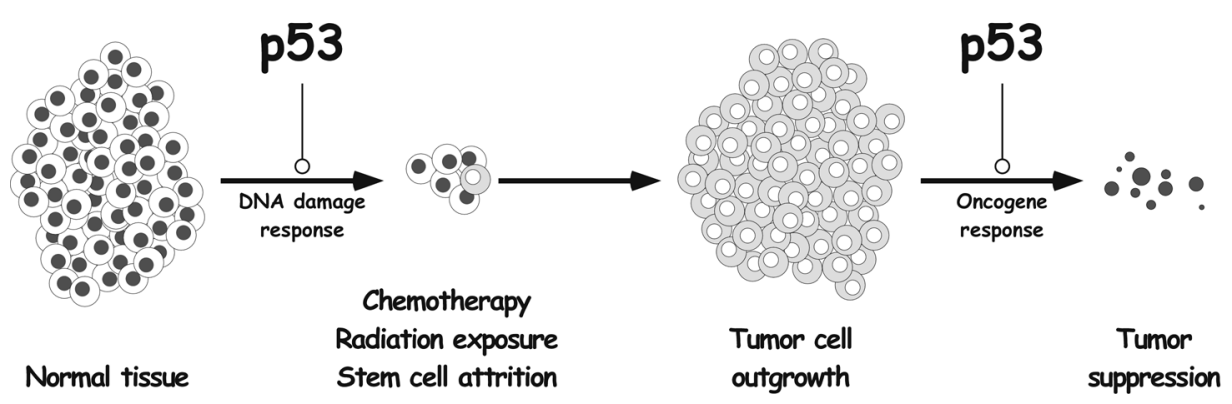

Figure 7. Schematic depiction of the two discrete putative roles of p53 in tumor suppression.

dundant, so it is probably not possible to block them all. An alternative strategy, however, might be to use pharmacological means to limit temporally p53 function throughout life. Our studies using the $p 53 E R^{T A M} K I$ mice indicate that only a relatively short burst of p53 function may be sufficient to cull incipient tumors, even long after the insult that initiated them. Hence, temporally restricting p53 function to short metronomic periods throughout life might provide sufficient periodic p53 function to staunch incipient tumors while offering substantial protection from DNA damage-induced pathologies in, say, patients receiving radio- or chemotherapy or astronauts sustaining chronic radiological exposure en route to Mars. A predominately "p53-free lifestyle" such as this might even ameliorate the otherwise ineluctable erosion of stem cell compartments and enhance longevity. Such notions are obviously very radical. However, the reversibly switchable $p 53 E R^{T A M} K I$ mouse model not only allows such ideas to be tested in principle, but also provides a test bed for fine-tuning the metronomic timing of p53 functionality so as to find the optimal balance between tumor suppression and radio resistance.

\section{CONCLUSIONS}

The rarity of spontaneous cancers is a strong clue that the evolutionary terrain available to somatic cells is severely constrained, limiting their autonomy and enforcing their obligatorily social nature. However, the mechanism by which such evolution is constrained remains an area of fierce debate. Switchable mouse models indicate that surprisingly small numbers of oncogenic mutation, when combined in highly specific, interdependent sets, carry all the information necessary to generate the full gamut of the neoplastic phenotype - a phenotype driven by the same programs that orchestrate and integrate developmental and regenerative expansion of normal tissues. In the future, such acutely switchable models will also serve as unique, in vivo preclinical test beds with which to establish not only which combinations of oncogenic lesions are required for the evolution and maintenance of cancers, but also what specific properties each type of lesion confers on the tumor phenotype. Knowing this will help direct interventions at the most effective targets for future cancer therapies.

\section{REFERENCES}

Askew D.S., Ihle J.N., and Cleveland J.L. 1993. Activation of apoptosis associated with enforced myc expression in myeloid progenitor cells is dominant to the suppression of apoptosis by interleukin-3 or erythropoietin. Blood 82: 2079.

Askew D., Ashmun R., Simmons B., and Cleveland J. 1991. Constitutive c-myc expression in IL-3-dependent myeloid cell line suppresses cycle arrest and accelerates apoptosis. Oncogene 6: 1915.

Campisi J. 2003. Cancer and ageing: Rival demons? Nat. Rev. Cancer 3: 339.

Christophorou M.A., Martin-Zanca D., Soucek L., Lawlor E.R., Brown-Swigart L., Verschuren E.W., and Evan G.I. 2005. Temporal dissection of $\mathrm{p} 53$ function in vitro and in vivo. Nat. Genet. 37: 718.

Coller H.A., Grandori C., Tamayo P., Colbert T., Lander E.S., Eisenman R.N., and Golub T.R. 2000. Expression analysis with oligonucleotide microarrays reveals that MYC regulates genes involved in growth, cell cycle, signaling, and adhesion. Proc. Natl. Acad. Sci. 97: 3260.

Eischen C.M., Weber J.D., Roussel M.F., Sherr C.J., and Cleveland J.L. 1999. Disruption of the ARF-Mdm2-p53 tumor suppressor pathway in Myc-induced lymphomagenesis. Genes Dev. 13: 2658.

Evan G. and Littlewood T. 1998. A matter of life and cell death. Science 281: 1317.

Evan G.I. and Vousden K.H. 2001. Proliferation, cell cycle and apoptosis in cancer. Nature 411: 342.

Evan G., Wyllie A., Gilbert C., Littlewood T., Land H., Brooks M., Waters C., Penn L., and Hancock D. 1992. Induction of apoptosis in fibroblasts by c-myc protein. Cell 63: 119.

Fernandez P.C., Frank S.R., Wang L., Schroeder M., Liu S., Greene J., Cocito A., and Amati B. 2003. Genomic targets of the human c-Myc protein. Genes Dev. 17: 1115.

Fukasawa K., Wiener F., Vande Woude G.F., and Mai S. 1997. Genomic instability and apoptosis are frequent in p53 deficient young mice. Oncogene 15: 1295.

Fukasawa K., Choi T., Kuriyama R., Rulong S., and Vande Woude G.F. 1996. Abnormal centrosome amplification in the absence of p53. Science 271: 1744.

Green D. and Evan G. 2002. A matter of life and death. Cancer Cell 1: 19

Guo Q.M., Malek R.L., Kim S., Chiao C., He M., Ruffy M., Sanka K., Lee N.H., Dang C.V., and Liu E.T. 2000. Identification of c-myc responsive genes using rat cDNA microarray. Cancer Res. 60: 5922.

Haggerty T.J., Zeller K.I., Osthus R.C., Wonsey D.R., and Dang C.V. 2003. A strategy for identifying transcription factor binding sites reveals two classes of genomic c-Myc target sites. Proc. Natl. Acad. Sci. 100: 5313.

Harrington E.A., Fanidi A., and Evan G.I. 1994a. Oncogenes and cell death. Curr. Opin. Genet. Dev. 4: 120.

Harrington E.A., Bennett M.R., Fanidi A., and Evan G.I. 1994b. c-Myc-induced apoptosis in fibroblasts is inhibited by specific cytokines. EMBO J. 13: 3286. 
Harvey M., Sands A.T., Weiss R.S., Hegi M.E., Wiseman R.W., Pantazis P., Giovanella B.C., Tainsky M.A., Bradley A., and Donehower L.A. 1993. In vitro growth characteristics of embryo fibroblasts isolated from p53-deficient mice. Oncogene 8: 2457.

Hermeking H. and Eick D. 1994. Mediation of c-Myc-induced apoptosis by p53. Science 265: 2091.

Inoue R., Asker C., Klangby U., Pisa P., and Wiman K.G. 1999. Induction of the human ARF protein by serum starvation. $\mathrm{An}$ ticancer Res. 19: 2939.

Kauffmann-Zeh A., Rodriguez-Viciana P., Ulrich E., Gilbert C., Coffer P., and Evan G. 1997. Suppression of c-Myc-induced apoptosis by Ras signalling through PI 3-kinase and PKB. $\mathrm{Na}$ ture 385: 544.

Land H., Parada L.F., and Weinberg R.A. 1983. Tumorigenic conversion of primary embryo fibroblasts requires at least two cooperating oncogenes. Nature 304: 596.

Lane D.P. 1992. Cancer. p53, guardian of the genome. Nature 358: 15.

Lowe S.W., Cepero E., and Evan G. 2004. Intrinsic tumour suppression. Nature 432: 307.

Mao D.Y., Watson J.D., Yan P.S., Barsyte-Lovejoy D., Khosravi F., Wong W.W., Farnham P.J., Huang T.H., and Penn L.Z. 2003. Analysis of Myc bound loci identified by CpG island arrays shows that Max is essential for Myc-dependent repression. Curr. Biol. 13: 882.

Menssen A. and Hermeking H. 2002. Characterization of the cMYC-regulated transcriptome by SAGE: Identification and analysis of c-MYC target genes. Proc. Natl. Acad. Sci. 99: 6274.

O'Connell B.C., Cheung A.F., Simkevich C.P., Tam W., Ren X., Mateyak M.K., and Sedivy J.M. 2003. A large scale genetic analysis of c-Myc-regulated gene expression patterns. J. Biol. Chem. 14: 14.
Pelengaris S., Khan M., and Evan G.I. 2002. Suppression of Myc-induced apoptosis in beta cells exposes multiple oncogenic properties of Myc and triggers carcinogenic progression. Cell 109: 321.

Rohn J.L., Hueber A.O., McCarthy N.J., Lyon D., Navarro P., Burgering B.M., and Evan G.I. 1998. The opposing roles of the Akt and c-Myc signalling pathways in survival from CD95-mediated apoptosis. Oncogene 17: 2811.

Van den Elsen P., Houweling A., and Van der Eb A. 1983. Expression of region $\mathrm{E} 1 \mathrm{~b}$ of human adenoviruses in the absence of region E1a is not sufficient for complete transformation. Virology 128: 377.

Vater C., Bartle L., Dionne C., Littlewood T., and Goldmacher V. 1996. Induction of apoptosis by tamoxifen-activation of a p53-estrogen receptor fusion protein expressed in E1A and T24 H-ras transformed $\mathrm{p} 53^{-1-}$ mouse embryo fibroblasts. Oncogene 13: 739.

Wagner A.J., Kokontis J.M., and Hay N. 1994. Myc-mediated apoptosis requires wild-type p53 in a manner independent of cell cycle arrest and the ability of $\mathrm{p} 53$ to induce $\mathrm{p} 21^{\text {waf } 1 / \mathrm{cip} 1}$. Genes Dev. 8: 2817.

Zeller K.I., Jegga A.G., Aronow B.J., O’Donnell K.A., and Dang C.V. 2003. An integrated database of genes responsive to the Myc oncogenic transcription factor: Identification of direct genomic targets. Genome Biol. 4: R69.

Zindy F., Eischen C.M., Randle D.H., Kamijo T., Cleveland J.L., Sherr C.J., and Roussel M.F. 1998. Myc signaling via the ARF tumor suppressor regulates p53-dependent apoptosis and immortalization. Genes Dev. 12: 2424.

Zindy F., Williams R.T., Baudino T.A., Rehg J.E., Skapek S.X., Cleveland J.L., Roussel M.F., and Sherr C.J. 2003. Arf tumor suppressor promoter monitors latent oncogenic signals in vivo. Proc. Natl. Acad. Sci. 100: 15930. 


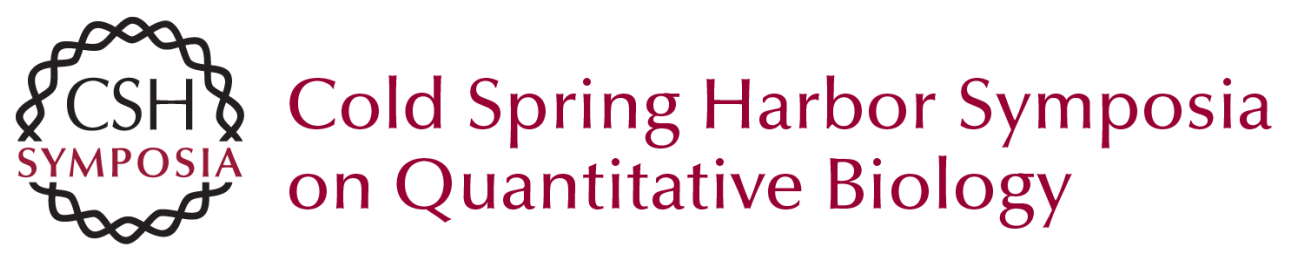

\section{Oncogene-dependent Tumor Suppression: Using the Dark Side of the Force for Cancer Therapy}

G.I. EVAN, M. CHRISTOPHOROU, E.A. LAWLOR, et al.

Cold Spring Harb Symp Quant Biol 2005 70: 263-273

Access the most recent version at doi:10.1101/sqb.2005.70.054

References This article cites 35 articles, 13 of which can be accessed free at: http://symposium.cshlp.org/content/70/263.full.html\#ref-list-1

License

Email Alerting Receive free email alerts when new articles cite this article - sign up in Service the box at the top right corner of the article or click here. 